\title{
WebTP : Les nouvelles technologies au service de l'aide à la préparation des travaux pratiques
}

\author{
G. Gateau, G. Fontes, N. Roux, F. Bonnet, F. Defay \\ Département Génie Electrique et Automatique ENSEEIHT - INPT \\ 2, rue Camichel, B.P. 7122 , 31071 Toulouse Cedex 7 FRANCE \\ Phone: +(33) 5615884 89, Fax: +(33) 05616388 75, mèl : Guillaume.Gateau@leei.enseeiht.fr
}

\begin{abstract}
RESUME Les travaux pratiques représentent de nos jours une part grandissante dans la formation de nos ingénieurs. C'est en particulier par cette forme d'enseignement très riche, que l'étudiant prend conscience des contraintes et réalités technologiques du domaine. La réduction des quotas horaires d'enseignement a conduit ces dernières années à une densification des travaux pratiques. Les textes s'enrichissent d'années en années, et bien souvent, effectuer le travail demandé dans le temps imparti devient presque impossible. A partir de ce constat peu optimiste, les auteurs exposent un projet d'aide à la préparation de travaux pratiques (TP) portant sur les thématiques de l'EEA, via le réseau Internet, destiné à des élèves ingénieurs. L'objectif est de proposer une approche originale et conviviale de la préparation des TP en s'appuyant sur des ressources (cours, méthodes expérimentales, matériel utilisé...). La préparation d'un TP consiste à naviguer dans ces ressources en suivant un chemin déterminé par l'enseignant, avec la libre possibilité d'approfondir les notions abordées plus ou moins directement dans le TP. La préparation sur Internet, effectuée en dehors des heures d'enseignements traditionnels donne lieu à un petit compte rendu écrit participant à l'évaluation globale du TP. Ce projet est l'occasion de mettre en place une base de ressources pour l'EEA. La première partie sera consacrée à l'introduction du projet puis nous présenterons dans la seconde partie les objectifs et le principe de fonctionnement de la préparation. La troisième partie est consacrée à la présentation du travail à effectuer par l'étudiant. La dernière partie sera réservée à la conclusion sur le projet.
\end{abstract}

Mots clés : enseignement, Aide à la préparation des travaux pratiques, Internet, présentation multimédia, Génie Electrique, WebTP.

\section{INTRODUCTION}

Le projet «WebTP », maintenant âgé de 5 ans, s'inscrit dans le cadre du développement de projets TICE (Technologie de l'Information et Communication pour l'Enseignement) de l'INP Toulouse. De nombreux projets et supports sont développés et proposés dans ce cadre mais très peu sont consacrés aux travaux pratiques qui représentent tout de même une large part de la formation voir même le lieu privilégié où se transmet le savoir et/ou le savoir faire.

C'est dans ce contexte que nous proposons depuis 4 ans un support d'aide à la préparation des travaux pratiques sur Internet. La mise en place est extrêmement longue et demande un travail très important pour reformater la présentation de ces travaux pratiques sous une forme exploitable sur Internet. De nombreuses personnes se sont succédées pour réaliser ce développement au cours de ces quatre dernières années incluant un enseignant chercheur permanent et huit doctorants ou ATER. Le projet arrive maintenant à maturité, c'est-àdire qu'une série complète de Travaux pratiques (destinés aux étudiants de première année Génie Electrique / Automatique) bénéficie de cette aide à la préparation. Cela représente $9 \mathrm{TP}$ et un nombre de pages assez conséquent.

Les travaux pratiques concernés par ce projet sont les travaux pratiques de type circuits destinés aux élèves ingénieurs de première année. D'une importance pri- mordiale, ils permettent, pour la majorité des étudiants, une découverte expérimentale de nombreuses disciplines de l'EEA comme l'Electronique analogique, l'Electrotechnique ou encore l'Electronique de puissance. Les séances de manipulation ont une difficulté progressive, adaptée à l'avancement des cours théoriques dispensés en parallèle.

L'évolution des technologies, des appareils de mesures, voire des méthodes et principes de mesures, est telle qu'aujourd'hui, les TP ont atteint une densité très importante, voire une certaine lourdeur. Ce projet, original et novateur, a pour objectif d'aérer et d'améliorer le déroulement global des TP en proposant aux étudiants une aide à la préparation de la manipulation expérimentale via le réseau Internet.

L'article sera organisé en deux parties suivies d'une conclusion. La première partie sera consacrée à la description et à la définition des objectifs principaux du projet. On détaillera ainsi le fonctionnement des travaux pratiques ainsi que l'architecture proposée sur le site pour l'aide à la préparation. La seconde partie sera quant à elle destinée à présenter ce que l'on pourrait appeler communément "le job de l'étudiant". Dans cette partie sera détailler ce que l'on attend de l'étudiant, les documents à rendre pour la préparation et bien entendu l'apport d'une telle approche sur la préparation et la réalisation des travaux pratiques. 


\section{OBJECTIFS ET ARCHITECTURE DU SITE}

\subsection{Descriptions et objectifs}

\subsubsection{Principes généraux du fonctionnement d'un TP}

Le cycle complet des travaux pratiques de type circuit s'étend sur un semestre et se compose de deux séries de TP tournants $(4+5)$, d'une séance de révision et d'une séance d'examen. La première série de 4 TP offre pour beaucoup d'étudiants une première approche à l'électrotechnique et à l'électronique. La difficulté est progressivement augmentée dans la seconde série de 5 TP.

Classiquement, chaque TP se divise en trois phases :

- Une première phase de préparation (hors salle) qui consiste en une lecture approfondie du texte exposant les notions théoriques utiles ainsi que le déroulement de la manipulation. Cette phase est individuelle et peut donner lieu à des calculs préliminaires, des analyses théoriques ...

- Une deuxième phase qui correspond à la séance de TP proprement dite (en salle). Durant ces 3 heures, les étudiants travaillent en trinôme.

- Et une dernière phase rédactionnelle qui rassemble les principaux résultats expérimentaux suivis d'une analyse dans un compte-rendu remis aux enseignants en fin de séance (en salle).

\subsubsection{Objectifs du projet "WebTP"}

Le projet «WebTP» s'inscrit dans la première phase d'un TP : la préparation. L'objectif premier de « Web » est de présenter une alternative à l'habituelle préparation écrite en essayant de rompre avec la platitude d'une formulation papier. Internet semble l'outil le mieux adapté à notre attente. Il offre de nouvelles perspectives pour la présentation et la préparation des TP. A travers l'interactivité qu'il propose, nous souhaitons rendre la préparation des TP plus attrayante pour l'étudiant.

Les acquis sont très différents suivant le parcours emprunté par l'élève : prépa $\mathrm{PC}, \mathrm{MP}$, prépa intégrée à l'INPT, IUT, STS,... Contrairement à un texte intégral, l'outil multimédia s'auto-adapte au niveau et au vécu de l'étudiant. Autour d'une ligne conductrice fixée par l'enseignant et retraçant les idées principales du TP, l'étudiant est libre d'approfondir ses connaissances via des mots-clés et/ou ressources cliquables.

Ce support apporte un véritable "outil" pédagogique grâce à l'utilisation d'applets java, de Gifs animés,... qui rendent les explications vivantes et conviviales. Pour satisfaire la curiosité de l'élève et lui proposer un approfondissement de ses connaissances, nous avons ajouté des liens vers d'autres sites Internet riches en informations. De plus, tout au long du parcours proposé, plusieurs questions sont disséminées permettant à l'étudiant d'évaluer son degré de compréhension du TP.

Le second objectif de «Web» est de simplifier au maximum la mise en œuvre des pages web. En effet, même si plusieurs logiciels permettent d'écrire assez simplement des pages HTML, la gestion et l'organisation d'un site Internet peuvent rapidement devenir longues et fastidieuses. Nous voulions rendre cette étape la plus simple et la plus transparente possible pour l'enseignant. La solution technologique retenue est Plei@d (prononcez Pleiad). Cette plateforme diffuse déjà les FOD (Formations Ouvertes à Distances) du CNAM depuis 1997.

La Figure 1 nous propose un synoptique du principe mis en avant pour le projet Web Un TP est donc divisé (partie haute) en une préparation hors salle et une traditionnel manipulation et rédaction d'un compte-rendu. Pour la phase de préparation (representée en dessous), l'étudiant devra se connecter au serveur de préparation (http://sudouest.pleiad.net) et sera ensuite en libre circulation sur le site entre présentations, étapes théoriques, étapes pratiques et fiches d'évaluation. Ces fiches de préparation sont essentielles et permettent à l'étudiant, au travers d'un petit devoir à rendre (dont les questions sont disséminées sur le site de préparation), d'évaluer sa préparation et donc de se poser les questions utiles avant d'arriver en séance.

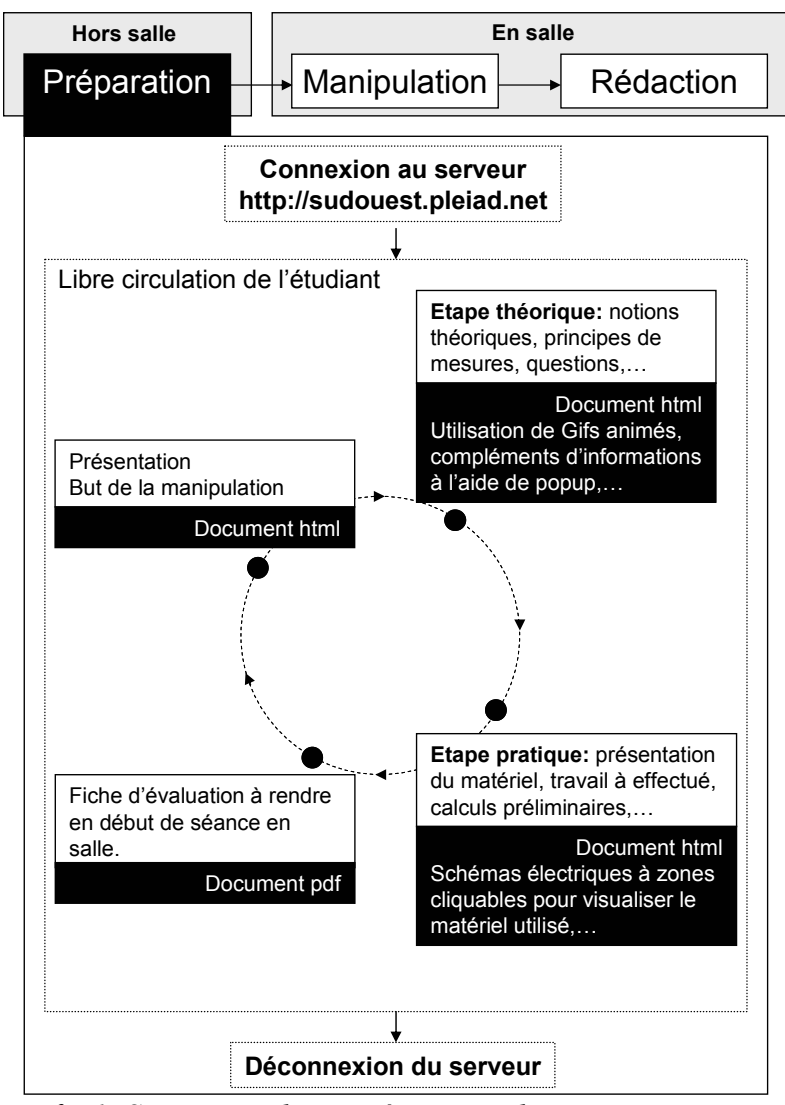

fig 1: Synoptique d'une préparation de TP sur Internet. 


\subsection{Architecture proposée du site}

Le site Internet CNAM propose une plate-forme, nommée Plei@d, et permettant de mettre en ligne des supports d'enseignement à distance (notes de cours, préparations de TP, exercices...). Le site Plei@d est géré par le CNAM et possède déjà sa propre architecture, en fonction de laquelle nous devons adapter la structure de Web

\subsubsection{Fonctionnement de la plate forme Plei@d}

Les sites de la plate forme Plei@d se divisent tout d'abord en unités d'enseignement (UE), elles mêmes divisées en différentes « séances », qui regroupent chacune un ensemble de "documents ». Ainsi, dans une unité d'enseignement, un administrateur peut créer autant de séances que nécessaire, et le nombre de documents voulus dans chaque séance.

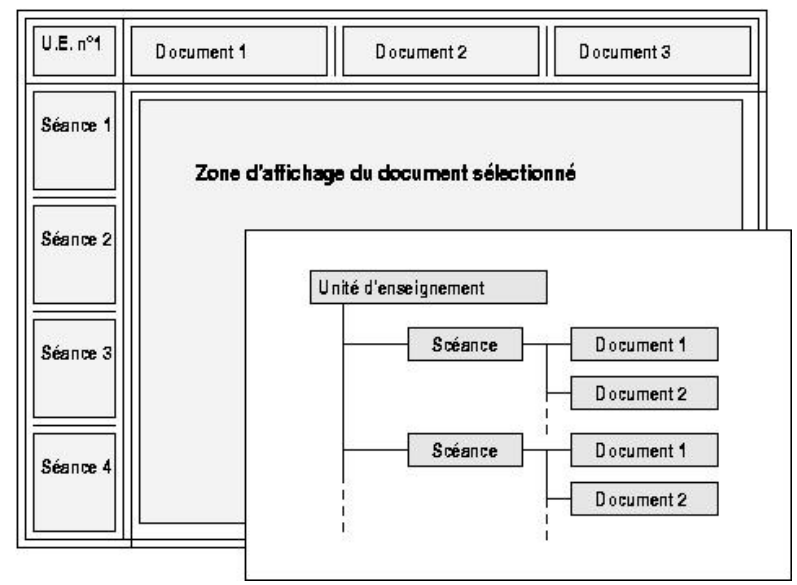

fig 2: Organisation de la plateforme Plei@de

Pour que l'étudiant puisse naviguer sans perdre le lien avec la structure de l'unité d'enseignement, le bandeau vertical situé à gauche de toutes les pages d'une même UE est constitué de liens vers ses différentes séances. De même, le bandeau horizontal situé en haut de page renvoie aux différents documents d'une séance. Lorsque l'on passe d'un document à l'autre, il s'affiche dans l'espace central de la page. Lorsque l'on passe d'une séance à l'autre, le bandeau supérieur se modifie en conséquence, et le premier document de la séance s'affiche dans la zone centrale. Le visiteur identifiant rapidement ces zones fixes du site, il peut revenir facilement à n'importe quelle séance ou document.

Quant à l'administration, une interface de gestion d'architecture est prévue par le CNAM : l'administrateur peut créer les séances et documents désirés en ligne, puis chaque document (en général une page web) doit être téléchargé indépendamment. Lors du téléchargement, les documents sont référencés automatiquement par le serveur du CNAM, et cette numérotation n'étant pas fixe, il est impossible de référencer un document dans un autre document par un lien hypertexte direct.
La structure de chaque TP doit donc être pensée en fonction de cette architecture particulière.

\subsubsection{Implantation de Web}

Pour Web, chaque TP sera une unité d'enseignement, toujours divisée en 5 séances:

- Introduction

- Etapes théoriques et préparation

- Préparation et prédétermination

- Fiches réponses

- Annexes.

Dans les deux premières sections, l'étudiant trouvera la théorie concernant directement le sujet du TP, la démarche expliquant le but de la manipulation, ainsi que quelques exercices d'applications; la section Préparation et prédétermination est consacrée à la mise en pratique de la théorie précédemment exposée, pour la détermination d'éléments directement utiles au TP (dimensionnement de composants, prédiction de comportement...). Les annexes regroupent quant à elles, les informations relatives au TP, telles que des mémos sur l'utilisation de certains appareils de mesure, ou sur l'application de méthodes particulières. Ces outils permettent à l'étudiant de prendre connaissance du contenu du TP, mais aussi d'élargir sa vision du contexte scientifique de la manipulation.

\section{LE JOB DE L'ETUDIANT}

Comme il a été décrit dans les paragraphes précédents, la mise en ligne des préparations de TP sur Internet ne se limite pas qu'à une simple diffusion électronique du texte de TP. L'étudiant se retrouve guidé dans sa préparation avec plus de convivialités et de moyens que ne permettent pas les supports papier.

\subsection{Apport de la préparation par Internet}

Comme nous le détaille la fig 1, l'étudiant découvre dans un premier temps les notions nécessaires à la réalisation du TP (rappels de cours, démonstrations, outils de calcul) et peut ainsi les relier directement à leurs applications concrètes.

Par exemple, il peut s'agir du rappel des outils de notation complexes comme présentée sur la fig 3, utilisés dans les applications en régime permanent sinusoïdal.

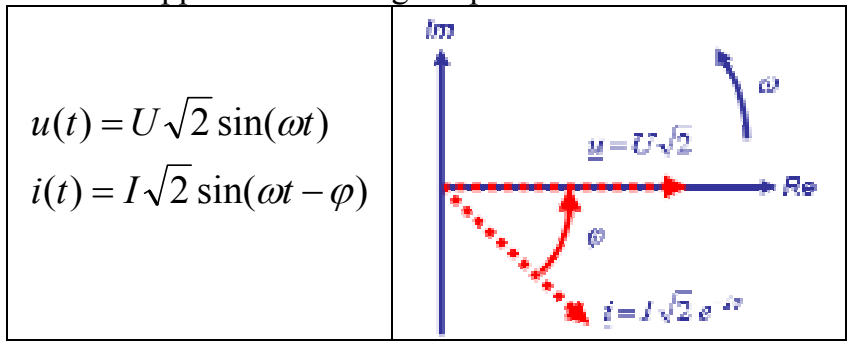

fig 3 : Représentation des grandeurs électriques complexes

Cette partie peut également faire l'objet de la présentation d'une application industrielle qui utilise le mon- 
tage vu pendant le TP. Par exemple dans le TP sur le redressement à thyristors, sont présentés le principe de la liaison continu IFA2000 France-Angleterre (fig 4), et les intérêts de la structure (la nécessité de la réversibilité est mise en évidence avec un tableau des imports/exports de consommation énergétique, le raccordement de deux réseaux alternatifs différents.)

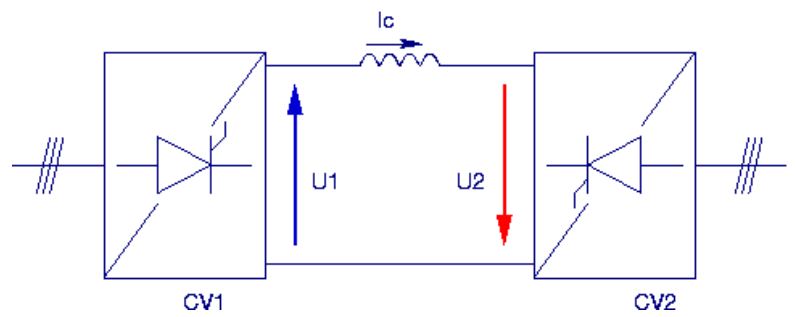

fig 4: Principe de la liaison continu France-Angleterre

Dans un second temps, l'étudiant découvre la manipulation à réaliser lors de la séance de TP. Il peut s'agir d'un développement sur le fonctionnement d'un appareil, sur une méthode de manipulation... Le support Internet permet ici de réaliser des présentations originales des plates-formes de manipulation, du matériel utilisé. Par exemple, les fonctionnalités de la plateforme d'alimentation du système triphasé sont mises en évidence ( fig 5, fig 6).

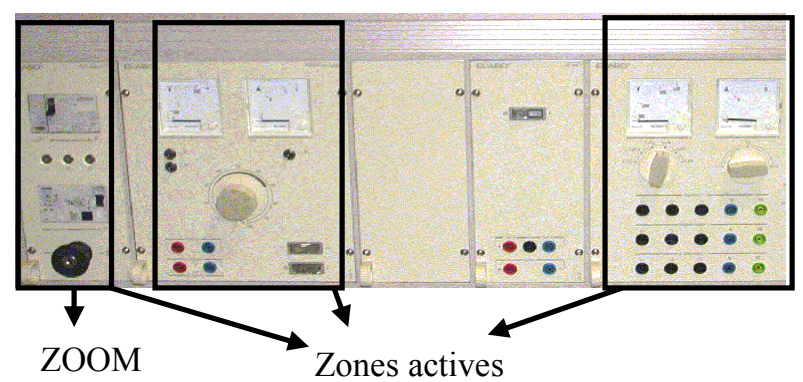

Figure suivante

fig 5 : Présentation globale de la plateforme d'alimentation

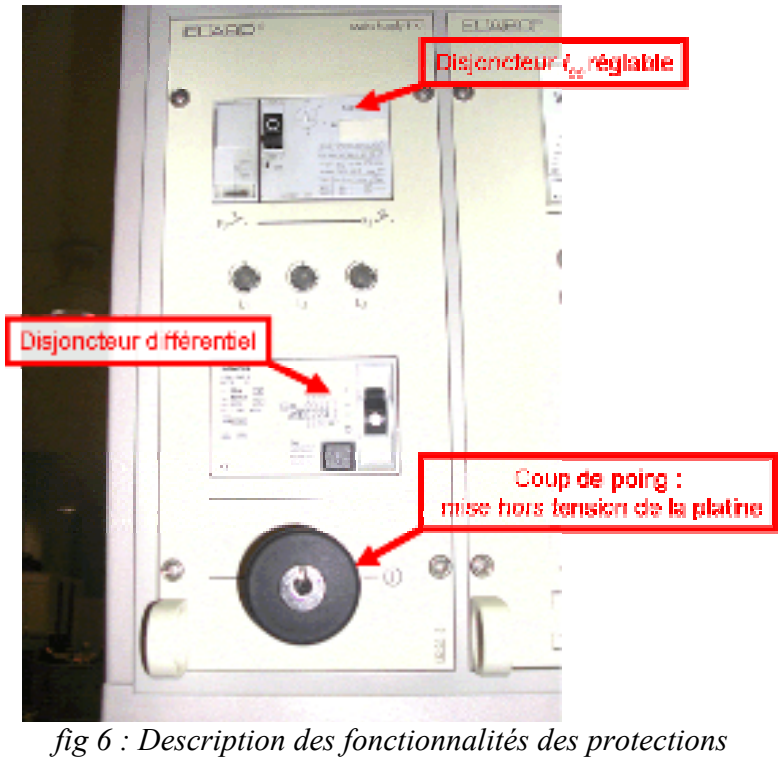

\subsection{Evaluation de l'étudiant}

Lors des différentes étapes, l'étudiant rencontre des questions et des exercices au fil des pages web. Ce travail fera l'objet d'un devoir écrit réalisé individuellement, à remettre en début de séance.

Dans l'étape théorique de la préparation d'un TP, l'étudiant peut être amené à refaire une démonstration qui constitue un point clé du TP. Par exemple, il peut s'agir de démontrer le principe de « la méthode des 2 Wattmètres » qui permet de mesurer des puissances dans un système électrique triphasé, ou bien de tracer les formes d'ondes d'un convertisseur statique.

Dans l'étape pratique, l'étudiant peut être amené à réfléchir sur un protocole de manipulation, ou dimensionner des éléments d'un montage qui lui serviront pendant le TP.

Ces divers exercices permettent à l'étudiant d'acquérir le vocabulaire et les techniques nécessaires pour discuter les points incompris avec l'enseignant, qui, connaissant les exercices de préparation, pourra structurer son discours en partant des points sur lesquels l'étudiant a déjà réfléchi. Cette réflexion personnelle de l'étudiant l'aidera à s'impliquer plus personnellement dans la réalisation du TP.

\section{CONCLUSIONS}

Ce projet représente une nouvelle façon d'aborder les $\mathrm{TP}$ de première année. Il permet d'accompagner les étudiants dans la préparation de travaux pratiques ayant souvent une complexité grandissante. Il fait appel à de nouveaux outils tels que le réseau Internet ou le multimédia, et permet aux étudiants de s'initier de manière attractive à l'EEA. Il ne s'agit en aucun cas de remplacer les TP traditionnels existants, mais bien de proposer une aide conviviale et interactive à la préparation. 
Le projet est déjà une réussite et fonctionne maintenant depuis 5 ans, une extension du projet aux autres années d'enseignement est envisagée. De la même façon, il est envisagé d'adapter ce type de projet à la formation continue où l'intérêt de l'aide à la préparation est là stratégique. La personne travaillant en entreprise peut ainsi préparer d'une façon beaucoup plus efficace ses passages en salles de TP.

Le développement de ce projet est également l'occasion de mettre en place une base de ressources pour l'EEA (matériels, cours et méthodes). Cette base de ressources étant générique, elle pourrait être utilisée dans d'autres expériences pédagogiques et illustrer nos disciplines en étant accessible sur Internet.

\section{Bibliographie}

1.G.Gateau, J.Regnier, JC. Hapiot, Etude du principe de fonctionnement d'un unite centrale de traitement CPU. In Proceedings of CETSIS Conf. (Nov, 2003, Toulouse), , pp. 173-174.

2.D. Hissel, D. Bouquain, A. Miraoui, Introduction aux systèmes pile à combustible. In Proceedings of CETSIS Conf. (Nov, 2003, Toulouse), , pp. 213216.

3. S. Mias. Outil "multimedia": une aide à l'approche fonctionnelle des systèmes. In Proceedings of CETSIS Conf. (Nov, 2003, Toulouse), , pp. 273-276.

4.C. Cubero-Castan. LILAS: une boite à outils pour la création d'appliquette de simulation logique. In Proceedings of CETSIS Conf. (Nov, 2003, Toulouse), , pp. 111-113.

\section{Remerciements}

Nous tenons à remercier l'INP Toulouse (TICE) qui a financé le développement de ce projet ainsi que toutes les personnes qui ont contribuées à son développement depuis le début. 